Document downloaded from:

http://hdl.handle.net/10251/123500

This paper must be cited as:

Lluesma-Rodriguez, F.; Hoyas, S.; Pérez Quiles, MJ. (2018). Influence of the computational domain on DNS of turbulent heat transfer up to $\mathrm{Re}-\mathrm{tau}=2000$ for $\operatorname{Pr}=0.71$. International Journal of Heat and Mass Transfer. 122:983-992.

https://doi.org/10.1016/j.ijheatmasstransfer.2018.02.047

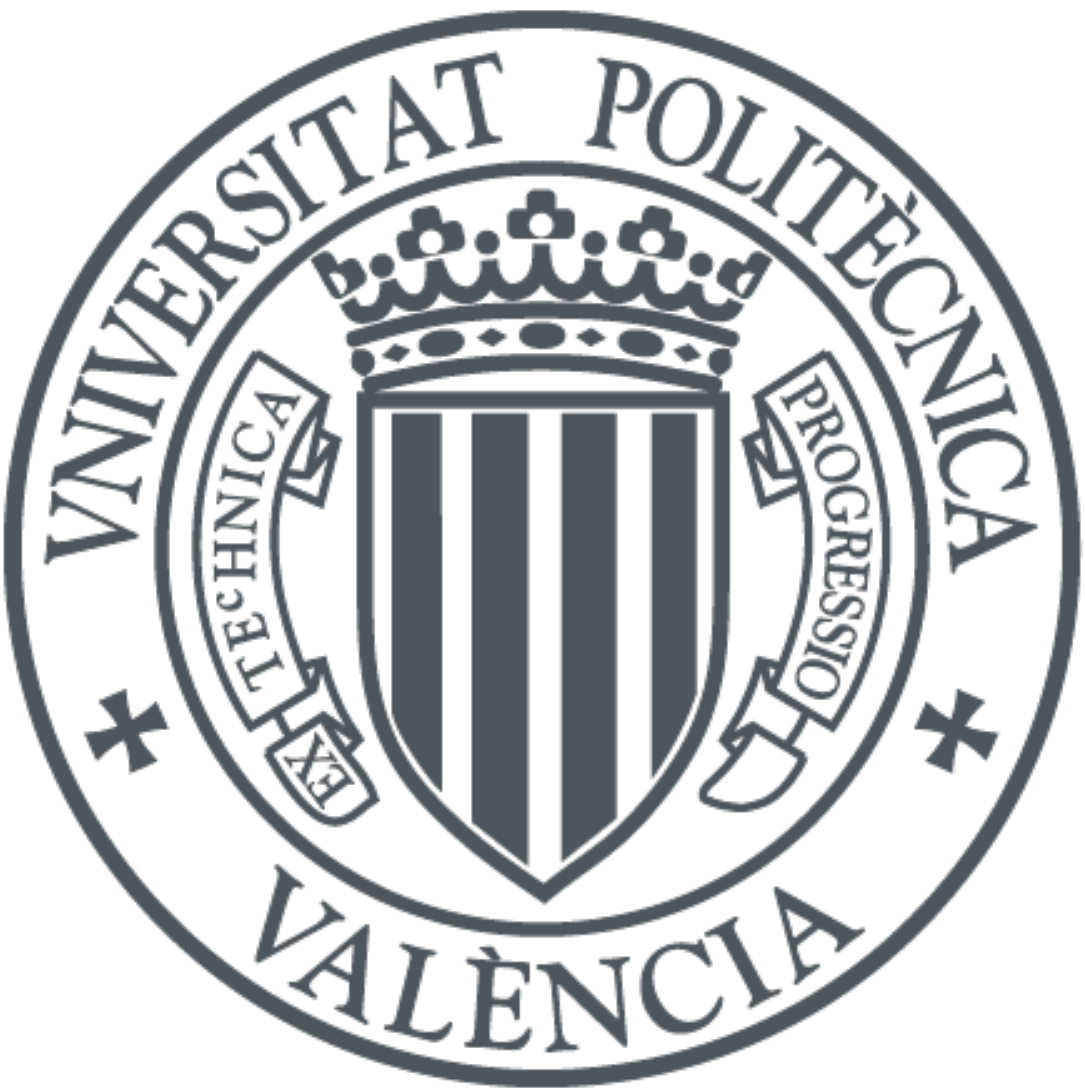

The final publication is available at

http://doi.org/10.1016/j.ijheatmasstransfer.2018.02.047

Copyright Elsevier

Additional Information 


\title{
Influence of the computational domain on DNS of turbulent heat transfer up to $R e_{\tau}=2000$ for $\operatorname{Pr}=0.71$
}

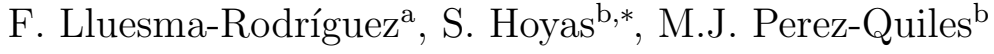 \\ ${ }^{a}$ ETS de Ingeniería del Diseño, Universitat Politècnica de València, València 46022, \\ Spain. \\ ${ }^{b}$ Instituto Universitario de Matemática Pura y Aplicada, Universitat Politècnica de \\ València, València 46022, Spain.
}

\begin{abstract}
We present a new set of direct numerical simulation data of a passive thermal flow in a turbulent plane Poiseuille flow with constant Prandtl number $\operatorname{Pr}=0.71$, and mixed boundary conditions. Simulations were performed at $R e_{\tau}=500,1000$, and 2000 for several computational domains in the range of $l_{x}=2 \pi h$ to $8 \pi h$ and $l_{z}=\pi h$ to $3 \pi h$. As a first key result we found that a length of $l_{x}=2 \pi h$ and a width of $l_{z}=\pi$ is enough to accurately obtain the one-point statistics and the budgets of the thermal kinetic energy, its dissipation and the thermal fluxes. None of them collapse exactly in wall units. On the other hand, the value of the thermal Kármán constant grows very slightly with the Reynolds number with a value of $\kappa_{t h}=0.44$ for $R e_{\tau}=2000$. The statistics of all simulations can be downloaded from the web page of our group.
\end{abstract}

Keywords: Turbulent channel flow, forced convection, Rayleigh number, Prandtl number

\section{List of symbols}

$x_{i} \quad$ Coordinate $x_{i}(\equiv x, y, z)$

$y \quad$ Vertical coordinate normalized with $h$ starting from 0

$U_{i} \quad$ Velocity in the direction $x_{i}$

$\Theta \quad$ Temperature

\footnotetext{
*Corresponding author. e-mail: serhocal@mot.upv.es. Tel: +34 - 963877007 (ext $76563)$
} 


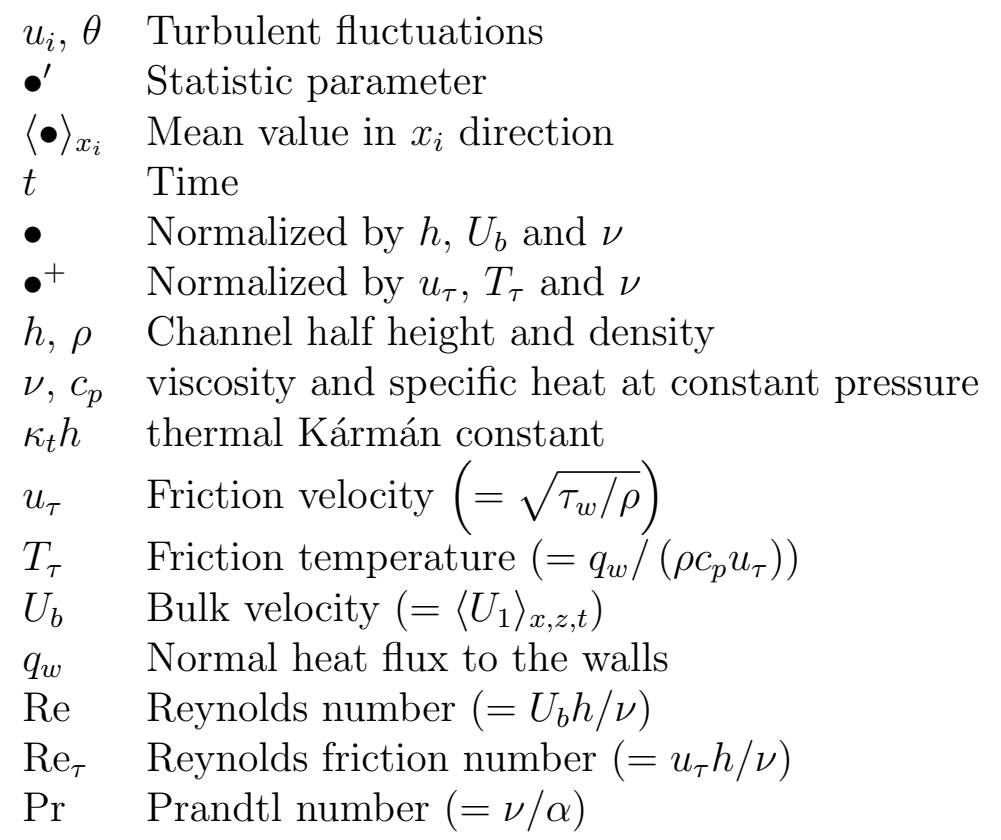

\section{Introduction}

The behavior of turbulent flows is still an open problem in physics and it is probably the one with most implications in day-to-day life. The study of the thermal properties of flows for different values of Reynolds, Prandtl and Rayleigh numbers are becoming more and more important. In a recent study for NASA, Slotnick et al [1] highlighted the importance of thermal flows in aeronautics applications for the foreseeable future. To cite only another example, for Prandtl number different to the one of air, a better knowledge of the dynamics of thermal flows is needed for the simulation of nuclear Liquid Metal Reactors (LMR) [2, 3]. Due to the continuous improvement of computer power, Direct Numerical Simulation (DNS) has become one of the most promising technique to study applications involving drag, noise or heat transmission. Moreover, as experiments involving thermal properties of turbulent wall flows are difficult to perform, DNS appears to be an essential tool.

The first DNS addressing heat transfer in turbulent channels was made by Kim and Moin in 1987 [4], for $R e_{\tau}=u_{\tau} h / \nu=180$ and $\operatorname{Pr}=0.71$. Here, $R e_{\tau}$ is the friction Reynolds number, $\operatorname{Pr}$ is the molecular Prandtl number, $u_{\tau}$ is the friction velocity, $h$ is the semi-height of the channel, and $\nu$ is the kinematic viscosity. Kim and Moin used an artificial boundary condition 
to describe the thermal flow, assuming constant heat generation throughout the fluid. This procedure has the advantage that this heat can be treated in the same way that the mean pressure gradient term is in the flow equations. Lyons et al [5] and Sumitani and Kasagi [6] used a different boundary condition, with walls at different temperature, without considering any effect of buoyancy. Kasagi et al [7] introduced a more realistic condition in 1992, named mixed boundary condition (MBC from now on). This condition was imposed on the two walls so that the local mean temperature increased linearly in the streamwise direction. With any buoyancy effect neglected, temperature was considered as a passive scalar. Using MBC as boundary condition, the Reynolds number has been increased constantly during the last few years for several Prandtl numbers. Saha et al [8], performed an excellent review of the different simulations made until very recently. Among these articles, it is worth to mention the work of Abe, Kawamura, and collaborators [9, 10]. Up to the knowledge of the authors, the largest DNS was made in 2004 by Abe et al [10], for $R e_{\tau}=1020$ and $\operatorname{Pr}=0.025$ and 0.71. More recently, Duponchel et al [2] made a Large Eddy Simulation at $R e_{\tau}=2000$, that is the largest $R e_{\tau}$ simulated up to now.

One critical point of DNS is the domain to be simulated. Saha et al in two papers [8, 11] investigated the length of the largest turbulent structures in pipes and the comparison between pipes and channels. In fact, in almost all of the papers cited in this introduction and Saha et al's works, the size of the computational box is narrower and shorter than in the classical turbulent channel flow simulations. While the more or less classical size of the computational domain for Poiseuille flows is $(8 \pi h, 2 h, 3 \pi h)$ [12, 13, 14, 15, 16], in most of the thermal flow simulations performed until now a box of length $4 \pi h$ has been used. It is well known that to accurately represent the largest structures of the flows, longer boxes are needed. Monty et al [17] found that the length of the largest meandering structures of wall flows is up to $25 \mathrm{~h}$. Moreover, in the case of Couette flows, the length of these structures is even longer, up to at least 80h, [18] demanding even larger domains.

However, one of the reasons for using short and narrow boxes is that DNS are computationally expensive. This cost can be approximated by $L_{x}^{2} L_{y} \operatorname{Re}_{\tau}^{4} \operatorname{Pr}^{3 / 2}$ [19], limiting the possibilities of running high Reynolds numbers. Lozano-Durán and Jiménez, [20] studying isothermal channel flows found that one-point statistics can be accurately computed in boxes with streamwise and spanwise sizes of only $2 \pi h \times \pi h$. In this paper we show that one-point statistic of the thermal flow, including dissipation and kinetic 
energy budgets, can be accurately represented in this box.

The paper is structured as follows. The numerical method and the validation strategy is explained in the second section. The statistics of the flow, including the turbulent budgets, are discussed in the third section. The turbulent structures of the flow are discussed in the fourth one. The fifth section contains the conclusions.

\section{Model description and formulation}

In this work, a new set of DNS of a passive thermal flow in a Poiseuille turbulent channel has been conducted within a computational box of $L_{x}=$ $\alpha \pi h, L_{y}=2 h$ and $L_{z}=\beta \pi h$, with spanwise and streamwise periodicity, and for several values of $\alpha$ and $\beta$.

The streamwise, wall-normal, and spanwise coordinates are $x, y$, and $z$ and the corresponding velocity components are $U, V$ and $W$ or, using index notation, $U_{i}$. The temperature is denoted by $T$ and the transformed temperature (see below) by $\Theta$. Statistically averaged quantities are denoted by an overbar, whereas fluctuating quantities are denoted by lowercase letters, i. e., $U=\bar{U}+u$.

The flow can be described by means of the momentum and mass balance equations, and the energy conservation principle. The fundamental equations for the fluid flow are the classical Navier-Stokes equations for incompressible flows [21].

$$
\begin{aligned}
\partial_{t} U_{i}^{+}+U_{j}^{+} \partial_{j} U_{i}^{+} & =-\partial_{j} P^{+}+\frac{1}{R e_{\tau}} \partial_{j j} U_{i}^{+} \\
\partial_{j} U_{j}^{+} & =0 .
\end{aligned}
$$

In these equations the superscript $(+)$ indicates that the quantities have been normalized by $u_{\text {tau }}$. As the thermal flow is modeled by the MBC, the averaged temperature grows linearly with respect to $x$. A transformed temperature $\Theta$ is introduced as $\Theta=T-T_{w}$ to satisfy the the constant heat flux boundary condition [9, 21]. The great advantage of this method is that $\Theta$ is periodic in the streamwise direction and Fourier methods can be applied. The equation for $\Theta$ becomes $([11])$,

$$
\partial_{t} \Theta^{+}+U_{j}^{+} \partial_{j} \Theta^{+}=\frac{1}{R e_{\tau} \operatorname{Pr}} \partial_{j j} \Theta^{+}+U_{1}^{+} /\left\langle U^{+}\right\rangle_{x y z}
$$




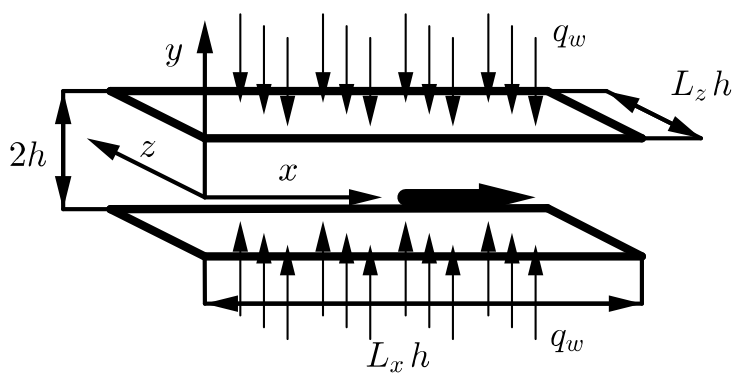

Figure 1: Sketch of the geometry and coordinate system

Here, the temperature has been adimensionalised by the friction temperature $T_{\tau}=q_{w} / \rho c_{p} u_{\tau}$. Using this model, the boundary conditions for the modified temperature are simply $\Theta(y=0,2 h)=0$.

Table 1 summarizes the parameters of the present simulations. The wallnormal grid spacing is adjusted to keep the resolution at $\Delta y=1.5 \eta$, i. e., approximately constant in terms of the local isotropic Kolmogorov scale $\eta=\left(\nu^{3} / \epsilon\right)^{1 / 4}$ for every $\operatorname{Re}_{\tau}$ case as in [14, 22]. In wall units, $\Delta y^{+}$varies from 0.42 at the wall up to $\Delta y^{+} \simeq 7.2$ at the centerline. At the center of the channel the resolutions along the three coordinates are approximately equal, $\eta-1.8 \eta$, [23]. The wall-parallel resolution is the one employed by Hoyas \& Jiménez [14] and similar to [16]. As $\operatorname{Pr}=0.7$, the thermal scales are expected to be of the same size that the ones of the flow, so the thermal flow does not need a grid refinement. The mesh sizes given in table 1 are comparable to the ones of Abe et al [10] for the streamwise direction and slightly better for the spanwise, as Abe et al use $\Delta_{z}^{+}=4.25$ for their $R e_{\tau}=1000$ case. Only the case P22 as a larger resolution, but without any effect on the convergence or the statistics (not shown). Cases P5 and P1 has been already computed, [10 but they have been computed again to validate the method used in this work, and to compare the different domains using a single method. Case P21 and P22 are completely new.

Equation 3 was implemented in LISO code, which has successfully been employed to run some of the largest simulations of turbulence [14, 24, 18, 22,. The Navier-Stokes equations are transformed into an equation for the wallnormal vorticity $\omega_{y}$ and for the Laplacian of the wall-normal velocity $\phi=\nabla^{2} v$ as in [12]. The spatial discretization uses dealiased Fourier expansions in $x$ and $z$ direction, and seven-point compact finite differences in $y$ direction with fourth-order consistency and extended spectral-like resolution [25]. The 


$\begin{array}{lcccccccccc}\text { Case } & \text { Line } & R e_{\tau} & L_{x} & L_{z} & N_{x} & N_{y} & N_{z} & \Delta x^{+} & \Delta z^{+} & T U_{b} / L_{x} \\ \text { P51 } & -\circ- & 500 & 2 \pi h & 1 \pi h & 384 & 251 & 384 & 8.18 & 4.09 & 588 \\ \text { P52 } & -*- & 500 & 4 \pi h & 2 \pi h & 768 & 251 & 768 & 8.18 & 4.09 & 200 \\ \text { P53 } & -\square- & 500 & 8 \pi h & 3 \pi h & 1536 & 251 & 1536 & 8.18 & 3.06 & 30 \\ \text { P11 } & -\circ- & 1000 & 2 \pi h & 1 \pi h & 768 & 383 & 768 & 8.18 & 4.09 & 18 \\ \text { P12 } & -*- & 1000 & 4 \pi h & 2 \pi h & 1536 & 383 & 1536 & 8.18 & 4.09 & 17.2 \\ \text { P13 } & -\square- & 1000 & 8 \pi h & 3 \pi h & 3072 & 383 & 2304 & 8.18 & 4.09 & 11 \\ \text { P21 } & -\circ- & 2000 & 2 \pi h & 1 \pi h & 1536 & 633 & 1536 & 8.18 & 4.09 & 30 \\ \text { P22 } & -*- & 2000 & 4 \pi h & 2 \pi h & 3072 & 633 & 2304 & 8.18 & 5.45 & 11\end{array}$

Table 1: Parameters of the simulations. Three different sets of friction Reynolds numbers are presented, in three different computational domains. $N_{x}, N_{y}, N_{z}$ are the numbers of collocation points. The last column denote the computational time span while statistics were taken in wash-outs $\left(U_{b} / L_{x}\right) . T$ is the computational time spanned by those fields. Line shapes given in the second column are used to identify the cases through almost all the figures of the present paper.

temporal discretization is a third-order semi-implicit Runge-Kutta scheme [26]. In every simulation, the flow had to evolve from an initial file, which has been taken from previous different simulations. The code was run until some transition phase has passed and the flow had adjusted to the new set of parameters. Once the flow is in a statistically steady state, statistics are compiled. The running times used to compile statistics are shown in the rightmost column of table 1. They are given in terms of wash-outs periods, where $U_{b}$ is the bulk velocity. The transitions until the simulations reached a statistically steady state, which can be very time consuming, are not contemplated in this table.

The validation of the code has been done in two separated steps. First, as equation 3 has been implemented in an already trusted code, the one point velocity statistics of the new database was compared to those previously obtained [14, 27] with the same code. The agreement was excellent, and it is not shown here for brevity. The second step was to validate the temperature field, using the data coming from the database of Kawamura, [9]. Figure 2 shows the very good agreement for the mean temperature, figure 2a, both in outer and inner scales. The thermal fluxes, and the rms of the temperature fluctuation can be seen in figure 2b. The agreement in both cases is almost perfect.

A further validation was made computing the total heat fluxes. Integrat- 


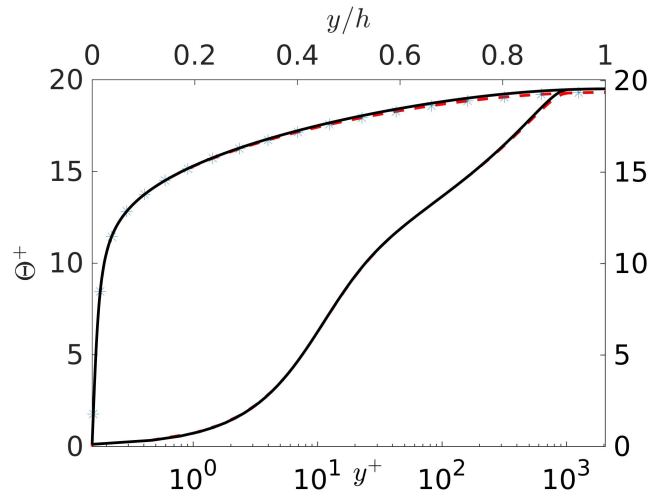

(a)

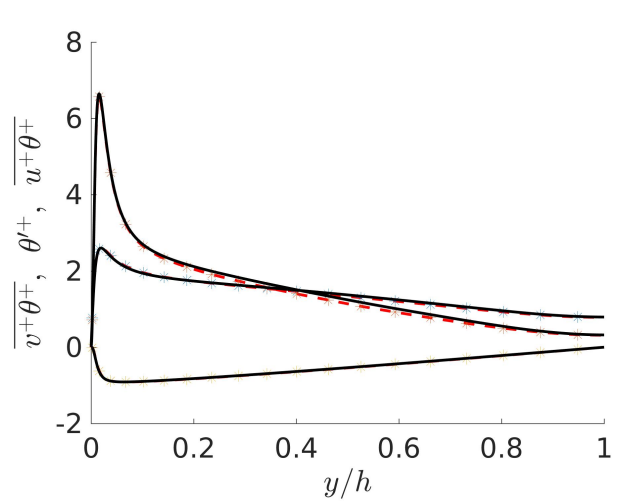

(b)

Figure 2: Colour online. (a) Mean temperature profile in inner (bottom) and outer (top) scales. (b) Wall normal and streamwise heat fluxes, and temperature RMS. Red dashed line, case P12 in Table 1. Black line from [9].

ing equation 3 one gets

$$
q_{\text {total }}^{+}=\frac{1}{\operatorname{Pr}} \frac{d \overline{\Theta^{+}}}{d y^{+}}-\overline{v^{+} \theta^{+}}=1-\frac{1}{R e_{\tau}} \int_{0}^{y^{+}} \frac{U_{1}^{+}}{\left\langle U^{+}\right\rangle} \mathrm{d} y^{+} .
$$

The total heat flux has been computed using the second and third terms of equation 4 and the difference between them is shown (red line) in the figure 3 for the cases P12 and P22. These results totally agree with those from 9. Moreover, for every case in the database this value was below $10^{-4}$, showing that enough statistics have been compiled.

\section{Statistics}

\subsection{Effect of the computational domain}

The mean temperature profiles are shown, for the whole database, in figure 4. They collapse fairly well to a logarithmic profile,

$$
\bar{\Theta}=\left(1 / \kappa_{t h}\right) \ln \left(y^{+}\right)+B
$$

which seems to be independent of the Reynolds number, figure 4 a, for $R e_{\tau} \geq$ 1000. The thermal Von Kármán constant, $\kappa_{t h}$ is $0.419,0.438$ and 0.440 for $R e_{\tau}=500,1000$ and 2000 respectively. This limit value agrees with the one computed by Abe et al [10]. The value of $B$ is approximately $B \approx 3.1$, 


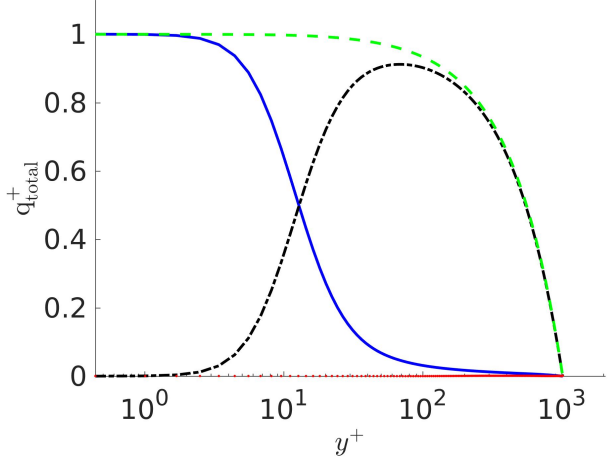

(a)

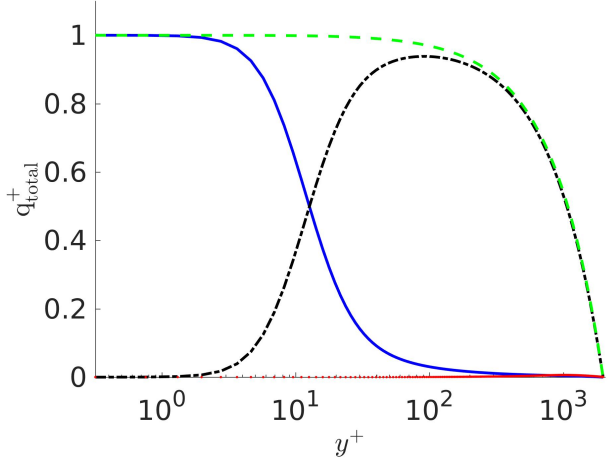

(b)

Figure 3: Colour online. Turbulent (black, dash-dot), molecular (blue) and total (green, dashed) heat fluxes. The red pointed line indicates the difference between the LHS and RHS of equation 4. (a) Case P12. (b) Case P22

close to the value obtained by Abe et al of $B=3.0$. The thermal diagnosis function defined as

$$
\Xi_{T}=y \partial_{y} \overline{\Theta^{+}}
$$

is shown in figure $4 \mathrm{~b}$. The agreement between the different domains for the same Reynolds number is remarkable along the whole channel for both figures. None of the cases presented here show a clear logarithmic profile, so simulations at larger friction Reynolds number are needed. There is an almost perfect collapse in the viscous layer below $y^{+}=50$, as in the case of the streamwise velocity [20, 22].

In figure 5, the root-mean-square (RMS) fluctuations of the temperature $\theta^{\prime+}=\left(\overline{\theta \theta}^{+}\right)^{1 / 2}$, and the thermal fluxes $\overline{u \theta}^{+}$and $\overline{v \theta}^{+}$are presented for the different Reynolds and domains. The independence of the results from the domain is also clear from figure 5, as all the cases collapse depending on their corresponding Reynolds number.

Neither the thermal fluxes nor the RMS of the temperature scale exactly in wall units near the wall. Furthermore, none of them presents a clear logarithmic law in the outer region. The maximum of these three statistics grows steadily with the Reynolds number. These maxima are at the same location in wall units, (not shown) stressing the fact that $L_{x} \approx 2 \pi$ and $L_{z} \approx \pi$ is enough to obtain good statistic across the whole channel. A last point in favor of this domain can be obtained from the turbulent Prandtl number, 


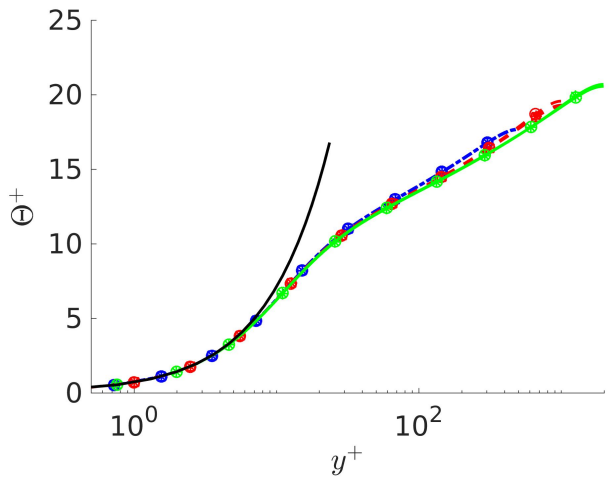

(a)

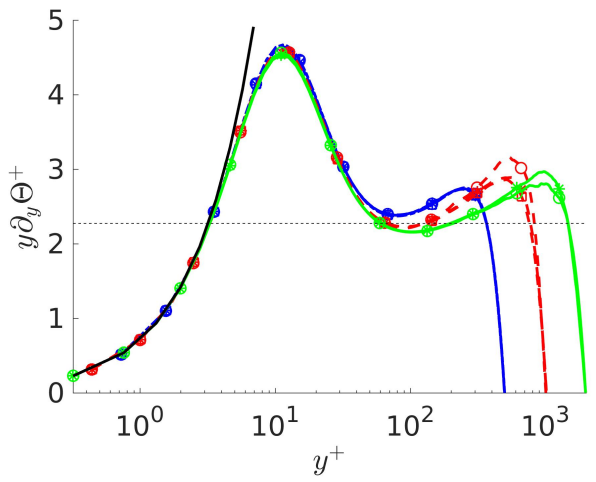

(b)

Figure 4: Colour online. Lines as in Table 1. (a) Mean temperature profile. (b) Thermal diagnostic function. In both figures, the black line is the thermal law of the wall, $\Theta=$ $\operatorname{Pr} y^{+}$. The dashed horizontal line corresponds to a thermal Von Kármán constant of 0.44.

which is defined as the ratio of the momentum eddy diffusivity $\nu_{t}$ to the thermal eddy diffusivity $\kappa_{t}$,

$$
\operatorname{Pr}_{t}=\frac{\nu_{t}}{\kappa_{t}}=\frac{\overline{u v}}{\overline{v \theta}} \frac{d \bar{\Theta} / d y}{d \bar{U} / d y} .
$$

Figure 5d shows $P r_{t}$ in the conductive sublayer, $\delta_{\theta}=\delta_{u} \operatorname{Pr}^{-1 / 3}$ [28], where $\delta_{u} \approx 6$ (fig. 4a). The values of $P r_{t}$ are similar to those of Kozuka et al [21], and the figure shows a small decrease in the value of $P r_{t}$ with $R e_{\tau}$. Furthermore, this figure shows that the smallest domain is enough to obtain good statistics even for quantities with quotients of derivatives in their definition.

\subsection{Heat fluxes, energy and dissipation budgets}

The budget equation for the component $\overline{u_{i} u_{j}}$ of the Reynolds stress tensor is given by Mansour et al [29] or Hoyas and Jimenez [24]. The interested reader is referred to these works and to Avsarkisov et al, [22] for further information in Poiseuille or Couette flows. Concerning thermal flows, the equation for the budgets of the turbulent heat fluxes, $\overline{u_{i} \theta}$, the temperature variance, $k_{\theta}=1 / 2 \theta^{2}$, and the dissipation rate of the temperature variance, $\varepsilon_{\theta}=\frac{1}{P r} \overline{\partial_{i} \theta \partial_{i} \theta}$ can be obtained from Sumitani and Kasagi [6] or Kozuka et al [21]. These equations are transcribed here to facilitate the reading of the paper. 


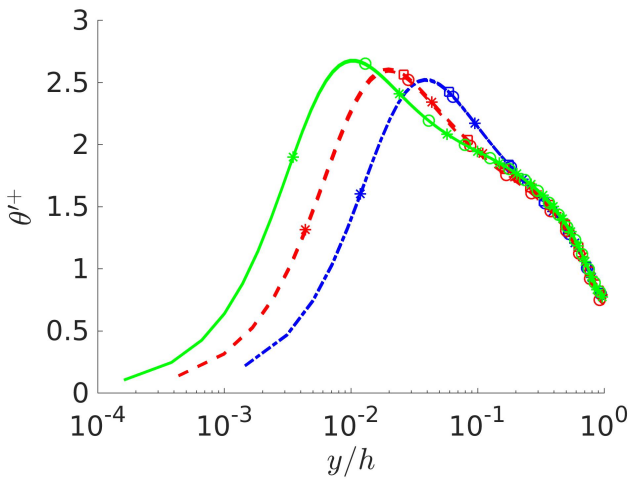

(a)

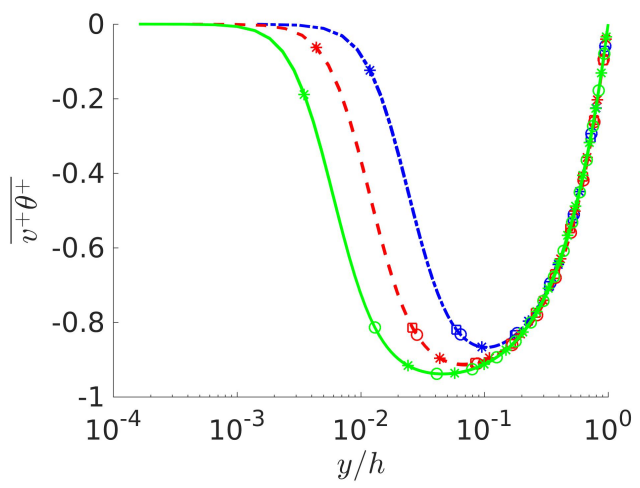

(c)

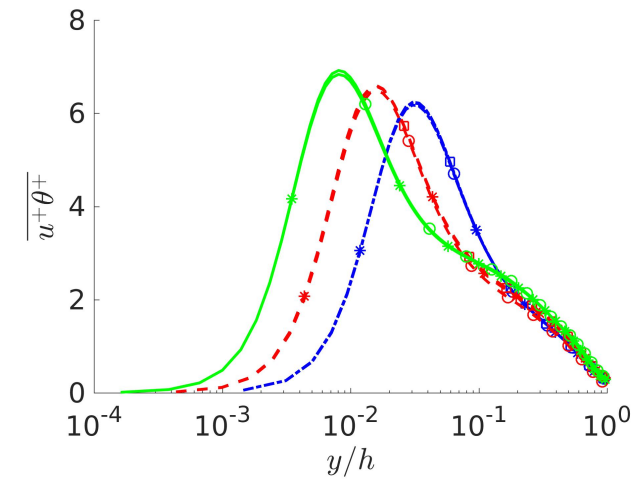

(b)

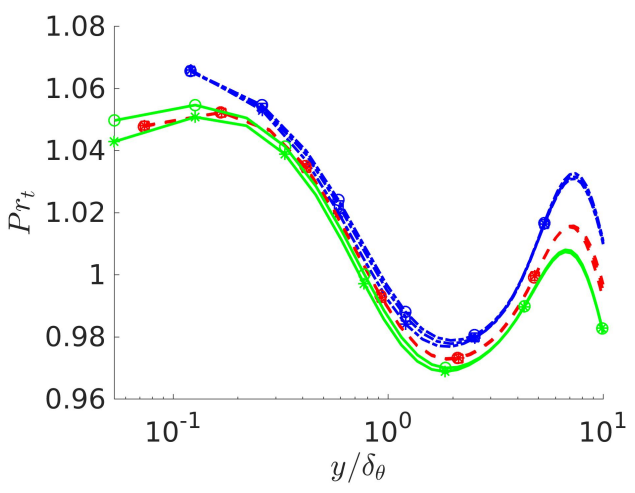

(d)

Figure 5: Colour online. (a) Intensity of the temperature fluctuation. Streamwise (b) and wall normal (c) heat fluxes. (d) Near-wall behaviour of the turbulent Prandtl number. Colours and lines as in Table 1 .

The budget equation for the turbulent heat fluxes $\overline{u_{i} \theta}$ is given by

$$
\frac{\overline{D u_{i} \theta}}{D t}=P_{i}+\varepsilon_{i}+T_{i}+\Pi_{i}^{s}+\Pi_{i}^{d}+V_{i}
$$

where $D / D t$ is the mean substantial derivative. The different terms on the right hand side are referred to as production, dissipation, turbulent diffusion, pressure-temperature gradient correlation, pressure diffusion, and viscous or molecular diffusion. They are respectively defined according to 


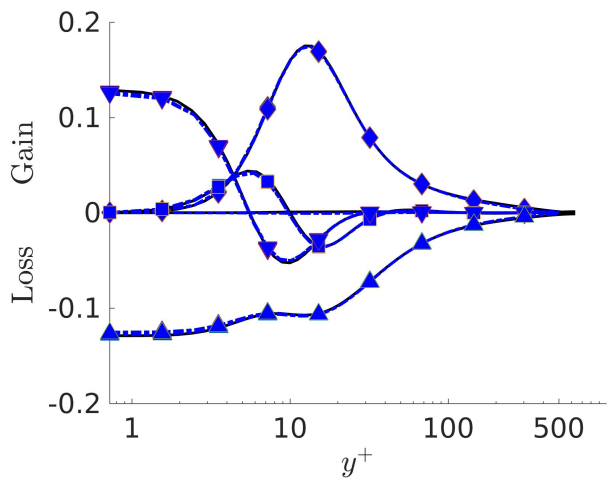

(a)

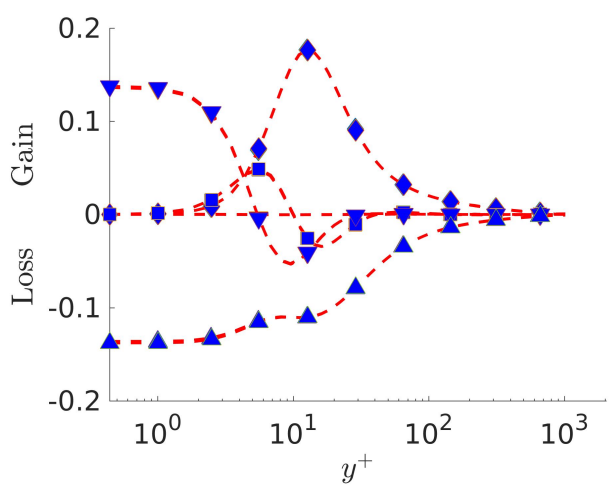

(c)

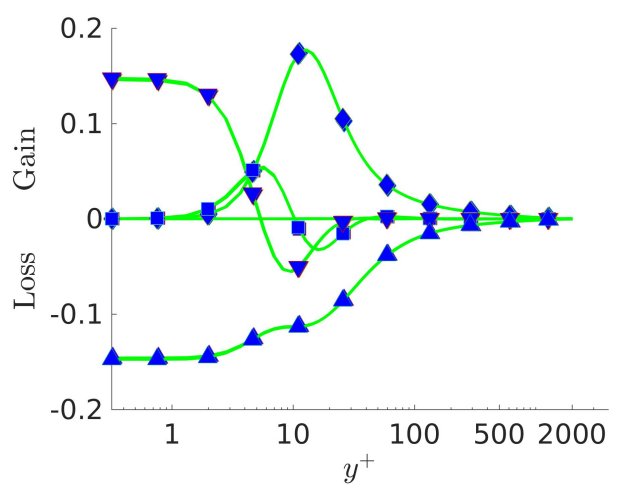

(e)

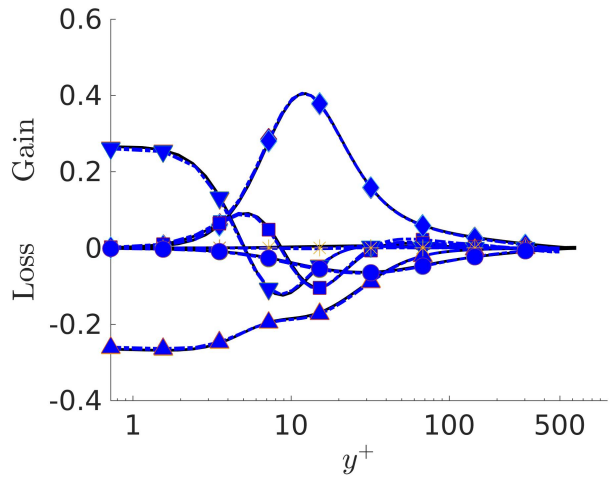

(b)

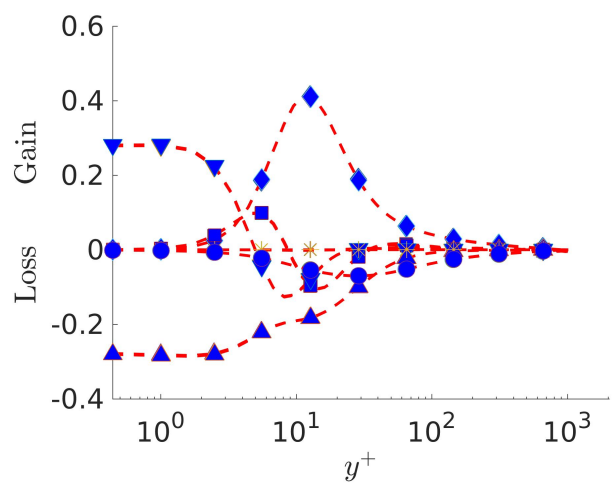

(d)

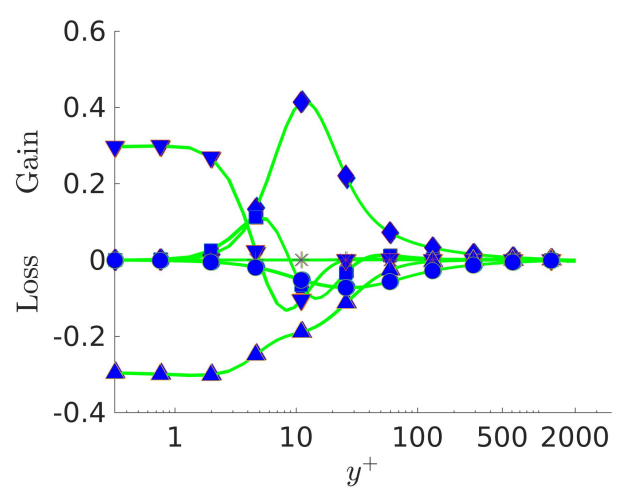

(f)

Figure 6: Colour online. Budgets of the temperature variance $k_{\theta}^{+}(\mathrm{a}, \mathrm{c}, \mathrm{e})$ and streamwise thermal flux (b, d, f) for the the three domains at $R e_{\tau}=500$ (a, b), $R e_{\tau}=1000$ (c, $\mathrm{d}$ ), and $R e_{\tau}=2000,(\mathrm{e}, \mathrm{f})$. Symbols change from table 1 Production $\diamond$, dissipation $\Delta$, viscous diffusion $\nabla$, pressure-strain $\circ$, pressure diffusion $*$, turbulent diffusion $\square$. Black line in (a, b), data from Kozuka et al 21$]$ 


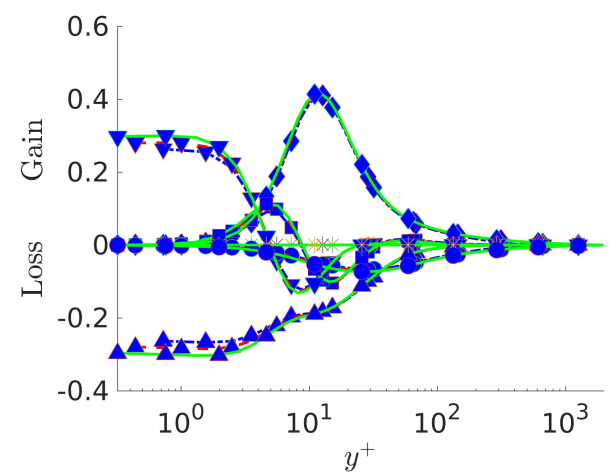

(a)

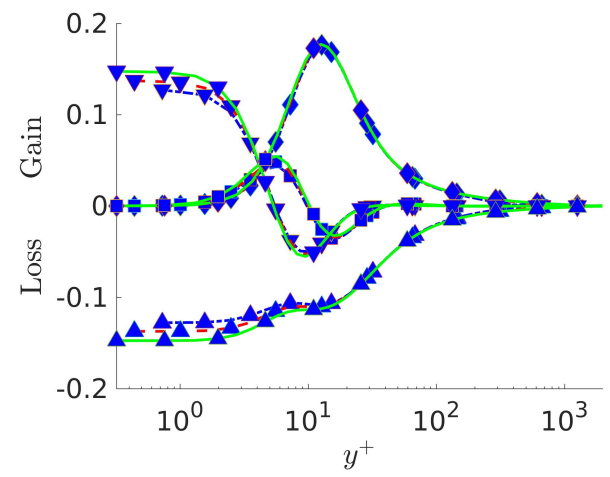

(c)

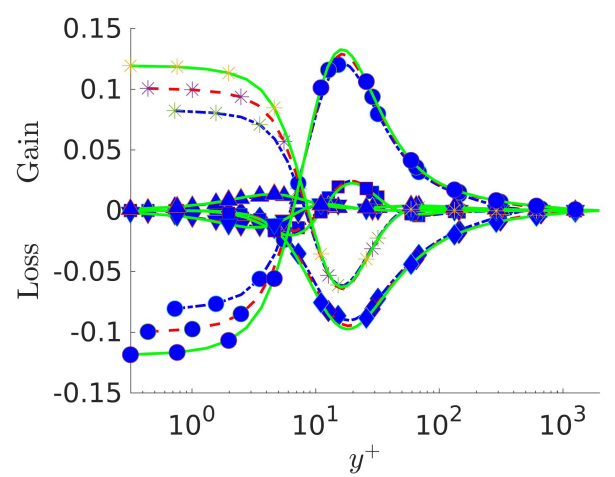

(b)

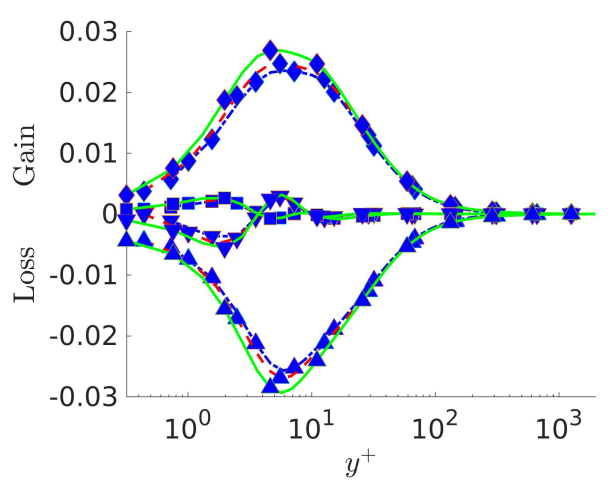

(d)

Figure 7: Colour online. Budgets of (a) Streamwise thermal flux $\overline{u \theta}$, (b) Normal heat flux $\overline{v \theta}$, (c) Temperature variance $k_{\theta}^{+}$, and (d) Dissipation, $\varepsilon_{\theta}$. Colours as in table 1, symbols changed. Production $\diamond$, dissipation $\Delta$, viscous diffusion $\nabla$, pressure-strain $\circ$, pressure diffusion $*$, turbulent diffusion $\square$. 


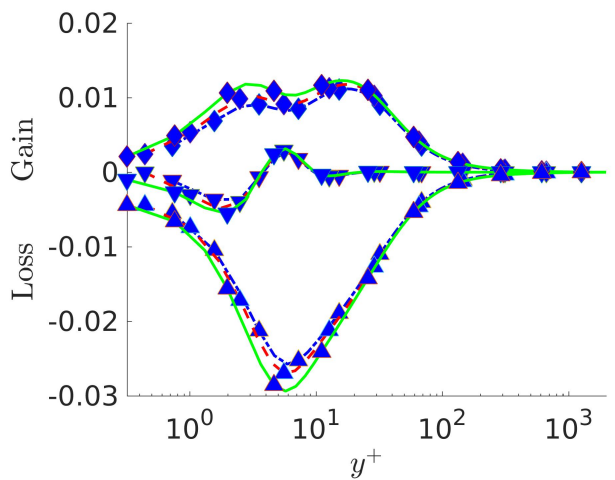

(a)

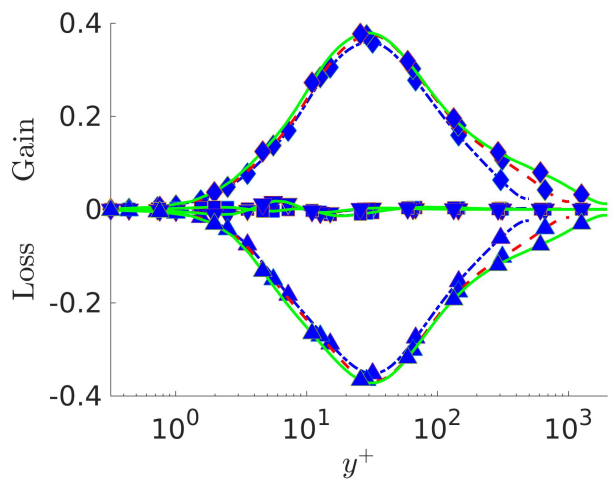

(b)

Figure 8: Colour online. Colours as in table 1, symbols changed. (a) Turbulent production $\diamond$, molecular diffusion $\nabla$, and dissipation $\Delta$, terms of the budgets for $\varepsilon_{\theta}$. These terms are the ones presenting the worst scaling. (b) Dissipation budget, scaled by $h \nu^{2} / u_{\tau}^{3} \theta_{\tau}^{2}$ and premultipled by $y^{+}$. The production terms have been sum up to facilitate the discussion. Symbols as in $7 \mathrm{~d}$ 


$$
\begin{aligned}
P_{i} & =-\overline{u_{i} v} \partial_{y} \bar{\Theta}-\overline{v \theta} \partial_{y} \overline{U_{i}} \\
\varepsilon_{i} & =-\nu\left(1+\frac{1}{P r}\right) \overline{\partial_{x_{k}} u_{i} \partial_{x_{k}} \theta}, \\
T_{i} & =-\partial_{x_{k}} \overline{u_{i} u_{k} \theta} \\
\Pi_{i}^{s} & =\overline{p \partial_{x_{i}} \theta} \\
\Pi_{i}^{d} & =-\partial_{x_{k}}\left(\delta_{k i} \overline{p \theta}\right), \\
V_{i} & =\nu \partial_{x_{k}}\left(\overline{\theta \partial_{x_{k}} u_{i}}+\frac{1}{P r} \overline{u_{i} \partial_{x_{k}} \theta}\right) .
\end{aligned}
$$

In the previous definitions, $\delta_{i j}$ is Kronecker's delta and repeated index imply summation over $k=1,2,3$. The transport equation of $k_{\theta}$ involves production, turbulent diffusion, viscous diffusion and dissipation terms,

$$
\frac{D \overline{k_{\theta}}}{D t}=P+T+V+\varepsilon_{\theta}
$$

These terms are defined as

$$
\begin{aligned}
P & =\overline{v \theta} \partial_{y} \bar{\Theta} \\
T & =-\frac{1}{2} \partial_{y} \overline{\theta^{2} v} \\
V & =\frac{1}{2 \operatorname{Pr}} \partial_{y y}^{2} \overline{\theta^{2}} \\
\varepsilon_{\theta} & =-\frac{1}{\operatorname{Pr}} \overline{\partial_{i} \theta \partial_{i} \theta}
\end{aligned}
$$

Finally, the transport equation for $\varepsilon_{\theta}$ is given by

$$
\frac{D \overline{\varepsilon_{\theta}}}{D t}=P_{m}+P_{m g}+P_{g}+P_{T}+T_{t}+V+\varepsilon_{\theta 1} .
$$

In this case, the terms are named mixed production, mean gradient production, gradient production, turbulent production, turbulent transport, molec- 
ular diffusion and dissipation.

$$
\begin{aligned}
P_{m} & =\frac{2}{\operatorname{Pr}} \overline{\partial_{i} v \partial_{i} \theta} \partial_{y} \bar{\Theta} \\
P_{m g} & =-\frac{2}{\operatorname{Pr}} \overline{\partial_{x} \theta \partial_{y} \theta} \partial_{y} \bar{U} \\
P_{g} & =-\frac{2}{\operatorname{Pr}} \overline{v \partial_{y} \theta} \partial_{y y}^{2} \bar{\Theta} \\
P_{t} & =-\frac{2}{P r} \overline{\partial_{i} \theta \partial_{j} \theta \partial_{j} u_{i}} \\
T_{t} & =-\frac{1}{\operatorname{Pr}} \partial_{y} \overline{v \partial_{i} \theta \partial_{i} \theta} \\
v_{\varepsilon_{\theta}} & =\frac{1}{\operatorname{Pr}^{2}} \partial_{y y}^{2} \varepsilon_{0}, \\
\varepsilon_{\theta 1} & =-\frac{2}{\operatorname{Pr}^{2}} \overline{\partial_{k j}^{2} \theta \partial_{k j}^{2} \theta}
\end{aligned}
$$

The effect of the domain in the budgets of the temperature variance and the streamwise thermal flux is shown in the figure 6 for all cases. This effect is hardly noticeable, as the different budgets collapse almost perfectly, even for $R e_{\tau}=2000$. To further validate the simulations, the budgets has been compared with those at the highest Reynolds available, 21], figures 6a and $6 \mathrm{~b}$. The agreement is excellent, both in the center of the channel and the wall.

As the effect of the domain is inappreciable, for the rest of the discussion the box $(4 \pi, 2 \pi)$ will be used. The four non-trivial budgets, for the three Reynolds numbers, are shown in the figure 7 . The data has been adimensionalised by $\nu / u_{\tau}^{3} \theta_{\tau}$ in the case of the thermal fluxes, figures $7 \mathrm{a}$ and $7 \mathrm{~b}$. For the kinetic energy, figure $7 \mathrm{c}$ the term $\nu / u_{\tau}^{2} \theta_{\tau}^{2}$ has been used. Finally, the dissipation, figure $7 \mathrm{~d}$, employs $\nu^{3} / u_{\tau}^{4} \theta_{\tau}^{2}$. In the latter figure, the four production terms have been sum up to facilitate the discussion.

The budgets of the heat fluxes, figures $7 \mathrm{a}$ and $7 \mathrm{~b}$, are similar to the budgets of $\overline{u u}$ and $\overline{u v}$ for isothermal flows [24, 22]. The budgets scale reasonably well beyond the sub-viscous layer, but again similarly to isothermal flows, the dissipation and the viscous diffusion terms do not scale close to the wall for $\overline{u \theta}$. The same problem arises for $\overline{v \theta}$ where the two pressure terms do not scale close to the wall. The budget of the kinetic energy shows the same problem. The situation is considerably worse in the budget for the $\varepsilon_{\theta}$, where 
the production of energy and the dissipation do not scale on the buffer layer. To further explore this scaling failure, figure $8 \mathrm{a}$ shows the terms presenting a worse scaling. These terms are the turbulent production, viscous diffusion and dissipation ones. Thus, any code modeling directly the thermal dissipation has to be extremely careful with the treatment of these terms. If taken into account that the viscous diffusion includes a second derivative at the wall, the lack of points in these region can produce severe errors. A second possible scaling for $\varepsilon_{\theta}$ is $h \nu^{2} / u_{\tau}^{3} \theta_{\tau}^{2}$. This scaling presents the advantage that, if premultiplied by $y^{+}$, equal areas under the curves means equal contribution. Using this scaling, the terms for the largest Reynolds numbers collapse, especially well in the buffer layer.

\section{Turbulent structures}

The instantaneous field of $\Theta$ is similar to the one of $U$. Figures $9 \mathrm{a}$ and $9 \mathrm{~b}$ show the $x y$ and $z y$ structure of the instantaneous thermal field. Large structures populate the center of the channel. The angle of the colder structures close to the wall in figure $9 \mathrm{a}$ is similar to those of the velocity field. Figure 9c shows the streak structure of the thermal flow close in the buffer layer. The center of the channel, figure $9 \mathrm{~d}$, presents longer structures, as it was expected.

Although the long and wide structures present in figure 9d could indicate that the domain is too short and narrow, the autocorrelation function confirms that the domain has been well chosen. The correlation length of the thermal disturbance at the center of the channel is shown in figure 10 . The two point autocorrelation coefficient $R_{\theta \theta}(\Delta x)$ is shown in figure 10a. For $x=2 \pi h, R_{\theta \theta}(\Delta x)$ is close to zero for all cases. Analogously, $R_{\theta \theta}(\Delta z)$, figure $10 \mathrm{~b}$, shows that domains with width less than $\pi h$ could not work due to a possible correlation of the data. It is also noticeable that the correlation length does not depend on the Reynolds number.

The spectral analysis definitively confirms that $l_{x}=2 \pi h$ and $l_{z}=\pi h$ are enough to accurately obtain the one-point statistics of the flow. In order to obtain further information about the turbulent structures of the flow, twodimensional spectral energy densities $\Phi=k_{x} k_{z} E\left(k_{x}, k_{z}\right)$ are used. Figure 11 shows the spectra of $\theta$ and the real part of the cospectra $u \theta$ at approximately $y^{+}=15$, the height of the near-wall kinetic-thermal-energy maximum.

Figure 11a shows the spectral densities for both $\theta$ and $u$. Two lines are given for each case, at 0.125 and 0.625 times the maximum of the spectrum. 


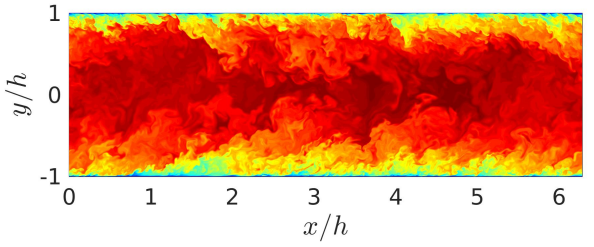

(a)

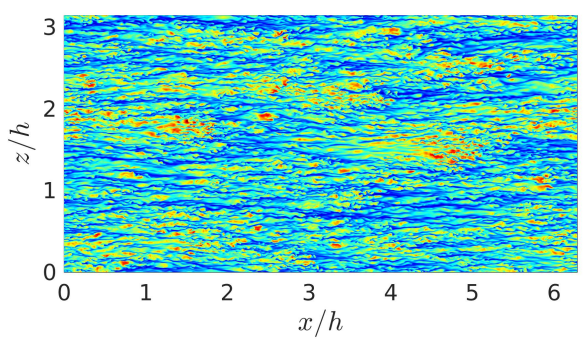

(c)

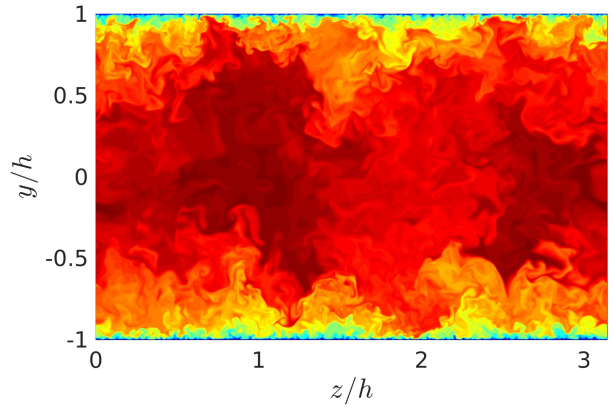

(b)

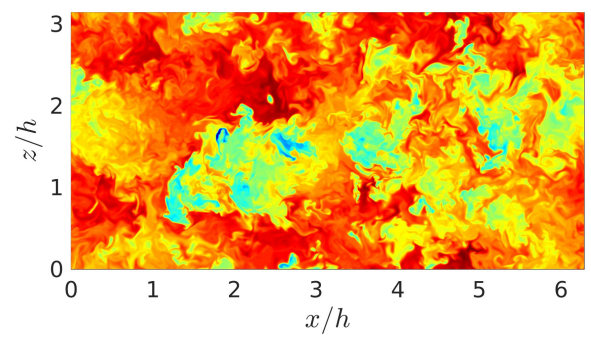

(d)

Figure 9: Colour online. Contours of the instantaneous $\Theta$ for the case C21. a) $y x$-plane. b) $z y$-plane. c) $x z$-plane at $y^{+} \approx 15$. d) $x z$-plane at the center of the channel

The maximum of the spectra are indicated by the symbol + . The spectra of the velocity, thin lines of figure 11a, collapse as the spectra of the $\theta$ does. Moreover, the maximum of both $u$ and $\theta$ are approximately at the same spot, indicating that the structures of the thermal field are highly correlated to those of the streamwise velocity. This can be confirmed from figure 11b. It can be seen that the cospectra and the $u$ spectra collapse almost perfectly. About the largest structures, the tail of the spectra indicates that the domain is filtering part of the energy of the flow. However, at least a $87.5 \%$ of the energy is well solved. This energy seems to no affect the statistics of the flow.

The situation is a bit different at the center of the channel. This can be appreciated in figure 12 where the spectral densities of $\Phi_{u u}$ (figure 12a) and 


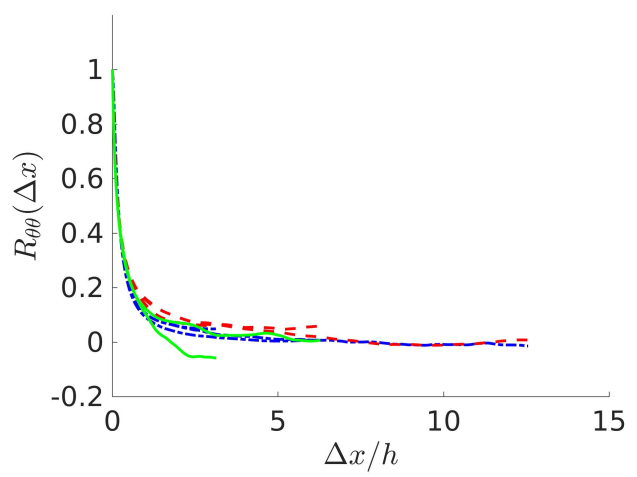

(a)

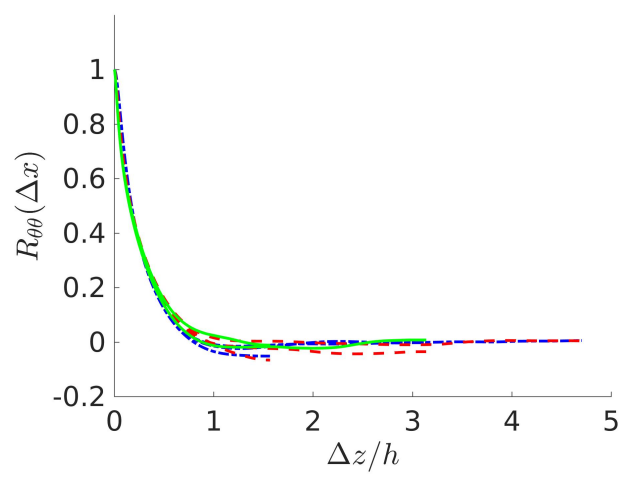

(b)

Figure 10: Colour online. Lines as in table 1. The two-point autocorrelation coefficient $R_{\theta \theta}$ of velocity fluctuations at the centerline, $y / h=1$ (a) Streamwise, (b) Spanwise.

$\Phi_{\theta \theta}(12 \mathrm{~b})$ are shown. Although the shape of both spectra is similar, a larger distribution of scales is seen in the thermal flow. Apart from this, figure 12 confirms the fact that no important information is lost in the smaller boxes. 


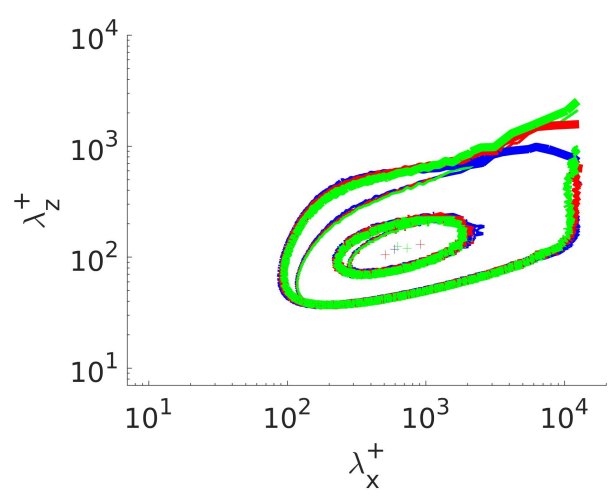

(a)

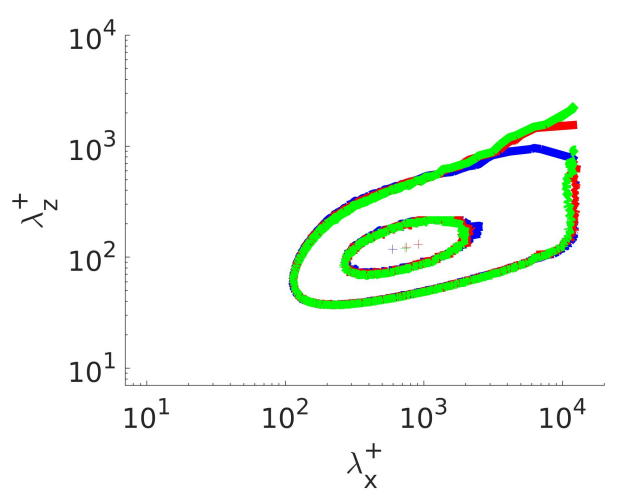

(b)

Figure 11: Colour online. Lines as in table 1. Spectral energy densities at $y \approx 15$, in terms of the wavelengths $\lambda=2 \pi / k$; (a) Thin lines, $\Phi_{u u}$, thick lines, $\Phi_{\theta \theta}$ (b)Thin lines, $\Phi_{u u}$, thick lines, real part of $\Phi_{u \theta}$. The spectra are normalized in wall units, and the two contours for each spectrum are 0.125 and 0.625 times the maximum of the spectrum.

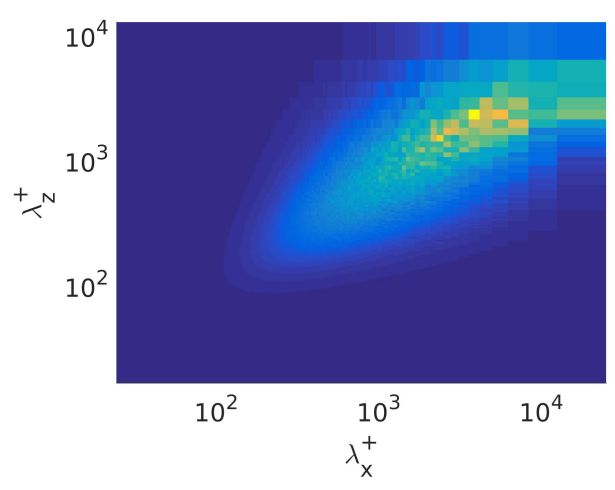

(a)

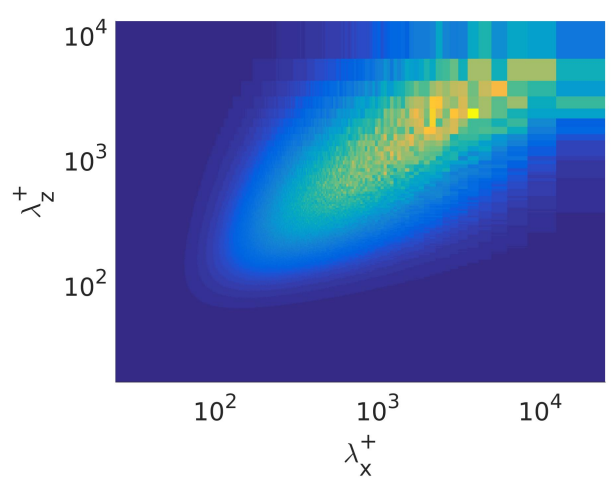

(b)

Figure 12: Colour online. Spectral energy densities at the center of the channel, in terms of the wavelengths $\lambda=2 \pi / k$; (a) $\Phi_{u u}$ (b) $\Phi_{\theta \theta}$. The spectra are normalized in wall units. 


\section{Conclusions}

In summary, seven new DNSs of heat transfer, at different Reynolds numbers, in turbulent channels have been presented. The simulations at $R e_{\tau}=500$ and $R e_{\tau}=1000$ have been used two-fold: first, to validate the implementation of the code. Second, to show that a relatively small computational domain of $(2 \pi, 2 h, \pi)$ have identical one-point statistics and turbulent budgets than longer and wider domains. This is true even if the largest structures do no fit in the domain.

Using the previous results, two more simulations for $R e_{\tau}=2000$ have been run. The results in the smallest domain were identical to the largest one. The comparison between the several simulations at different Reynolds numbers shows several scaling problems. Neither the rms of the temperature nor the thermal fluxes scale properly. It has been impossible to identify any logarithmic law for these quantities. As in the case of the streamwise velocity, the indicator function shows that the logarithmic layer is still not totally present. Also similarly to isothermal flows, the turbulent budgets present several scaling failures. The most serious ones occur in the budgets

for $\overline{v \theta}$ and the dissipation $\varepsilon_{\theta}$. An alternative scaling shows a better behaviour for the latter.

The analysis of the structures shows that the length and width of the thermal structures scale well in wall units for the different Reynolds numbers. This scales are similar to those of the streamwise velocity. Apart from visual identification from instantaneous images of $U$ and $\Theta$, this fact has been confirmed through the spectra of $U$ and the cospectra of $U$ and $\Theta$.

\section{Acknowledgments}

This work was supported by the Ministerio de Empleo y Competitividad, project ENE2015-71333-R. The computations of the new simulations were made possible by a generous grant of computing time from the Supercomputation center of the Universitat Politècnica de València. We are grateful to Mr. Kawamura for providing us with copies of their original data.

\section{References}

[1] J. Slotnick, A. Khodadoust, J. Alonso, D. Darmofal, W. Gropp, E. Lurie, and D. Mavriplis. Cfd vision 2030 study: A path to revolutionary 
computational aerosciences. NASA TECHNICAL REPORT, (218178), 2014.

[2] M. Duponcheel, L. Bricteux, M. Manconi, G. Winckelmans, and Y. Bartosiewicz. Assessment of rans and improved near-wall modeling for forced convection at low prandtl numbers based on les up to $R e_{\tau}=2000$. International Journal of Heat and Mass Transfer, 75:470-482, 2014.

[3] G. Grötzbach. Challenges in low-prandtl number heat transfer simulation and modelling. Nuclear Engineering and Design, 264:41-55, 2013.

[4] John Kim and Parviz Moin. Transport of passive scalars in a turbulent channel flow. NASA, 1(TM-89463):1-14, 1987.

[5] S.L. Lyons, T.J. Hanratty, and J.B. McLaughlin. Direct numerical simulation of passive heat transfer in a turbulent channel flow. International Journal of Heat and Mass Transfer, 34(4-5):1149-1161, 1991. cited By 100.

[6] Y. Sumitani and N. Kasagi. Direct numerical simulation of turbulent transport with uniform wall injection and suction. AIAA Journal, 33:1220-1228, 1995.

[7] N Kasagi, Y Tomita, and A Kuroda. Direct numerical simulation of passive scalar field in a turbulent channel flow. Journal of Heat Transfer, 114(3):598-606, August 1992.

[8] S Saha, Cheng Chin, HM Blackburn, and ASH Ooi. The influence of pipe length on thermal statistics computed from DNS of turbulent heat transfer. International Journal of Heat and Fluid Flow, 32(6):1083-1097, 2011.

[9] Hiroshi Kawamura, Kouichi Ohsaka, Hiroyuki Abe, and Kiyoshi Yamamoto. DNS of turbulent heat transfer in channel flow with low to medium-high Prandtl number fluid. International Journal of Heat and Fluid Flow, 19(5):482-491, 1998.

[10] Hiroyuki Abe, Hiroshi Kawamura, and Yuichi Matsuo. Surface heatflux fluctuations in a turbulent channel flow up to $R e_{\tau}=1020$ with $\operatorname{Pr}=0.025$ and 0.71. International Journal of Heat and Fluid Flow, 25(3):404-419, 2004. 
[11] S. Saha, J.C. Klewicki, A.S.H. Ooi, and H.M Blackburn. Comparison of thermal scaling properties between turbulent pipe and channel flows via dns. International Journal of Thermal Sciences, 89:43-57, 2015.

[12] J. Kim, P. Moin, and R. Moser. Turbulence statistics in fully developed channels flows at low Reynolds numbers. Journal of Fluid Mechanics, 320:259-285, 1987.

[13] R.D. Moser, J. Kim, and N.N. Mansour. Direct numerical simulation of turbulent channel flow up to $R e_{\tau}=590$. Physics of Fluids, 11(4):943945, 1999.

[14] Sergio Hoyas and Javier Jiménez. Scaling of the velocity fluctuations in turbulent channels up to $R e_{\tau}=2003$. Physics of Fluids, 18(1):011702, 2006 .

[15] M. Bernardini, S. Pirozzoli, and P. Orlandi. Velocity statistics in turbulent channel flow up to $R e_{\tau}=4000$. Journal of Fluid Mechanics, 758:327343, 2014.

[16] M. Lee and R. Moser. Direct numerical simulation of turbulent channel flow up to $R e_{\tau} \approx 5200$. Journal of Fluid Mechanics, 774:395-415, 2015.

[17] J.P. Monty, J.A. Stewart, R.C. Williams, and M.S. Chong. Large-scale features in turbulent pipe and channel flows. Journal of Fluid Mechanics, $25: 147-156,2007$.

[18] V. Avsarkisov, S. Hoyas, M. Oberlack, and J.P. García-Galache. Turbulent plane Couette flow at moderately high reynolds number. Journal of Fluid Mechanics, 751:R1, 2014.

[19] T. Yano and N Kasagi. Direct numerical simulation of turbulent heat transport at high prandtl numbers. JSME International Journal, Series B: Fluids and Thermal Engineering, 42(2):284-292, 1999.

[20] A. Lozano-Durán and J. Jiménez. Effect of the computational domain on direct simulations of turbulent channels up to $R e_{\tau}=4200$. Physics of Fluids, 26(1):011702, 2014.

[21] Makoto Kozuka, Yohji Seki, and Hiroshi Kawamura. DNS of turbulent heat transfer in a channel flow with a high spatial resolution. International Journal of Heat and Fluid Flow, 30(3):514-524, 2009. 
[22] V. Avsarkisov, M. Oberlack, and S. Hoyas. New scaling laws for turbulent Poiseuille flow with wall transpiration. Journal of Fluid Mechanics, 746:99-122, 2014.

[23] A.J. Smits, J. Monty, M. Hultmark, S.C.C. Bailey, N. Hutchins, and I. Marusic. Spatial resolution correction for wall-bounded turbulence measurements. Journal of Fluid Mechanics, 676:41-53, 2011. cited By 60 .

[24] S. Hoyas and J. Jiménez. Reynolds number effects on the Reynoldsstress budgets in turbulent channels. Physics of Fluids, 20(10):101511, 2008 .

[25] S. K. Lele. Compact finite difference schemes with spectral-like resolution. Journal of Computational Physics, 103(1):16-42, 1992.

[26] Philippe R Spalart, Robert D Moser, and Michael M Rogers. Spectral methods for the Navier-Stokes equations with one infinite and two periodic directions. Journal of Computational Physics, 96(2):297-324, 1991.

[27] Javier Jiménez and Sergio Hoyas. Turbulent fluctuations above the buffer layer of wall-bounded flows. Journal of Fluid Mechanics, 611:215236, 2008.

[28] D.A. Shaw and T.J. Hanratty. Turbulent mass transfer rates to a wall for large schmidt numbers. AIChE Journal, 23(1):28-37, 1977. cited By 146.

[29] N. N. Mansour, J. Kim, and P. Moin. Reynolds-stress and dissipationrate budgets in a turbulent channel flow. Journal of Fluid Mechanics, 194:15, 1988. 